\title{
Instability analysis of torsional MEMS/NEMS actuators under capillary force
}

\author{
Jian-Gang Guo ${ }^{\text {a }}$, Li-Jun Zhou ${ }^{\text {b }}$, Ya-Pu Zhao ${ }^{\text {c,* }}$ \\ a Department of Mechanics, Tianjin University, Tianjin 300072, People's Republic of China \\ ${ }^{\mathrm{b}}$ Department of Mechanical Engineering, Tianjin University of Technology and Education, Tianjin 300222, People's Republic of China \\ c State Key Laboratory of Nonlinear Mechanics, Institute of Mechanics, Chinese Academy of Sciences, Beijing 100190, People's Republic of China
}

\section{A R T I C L E I N F O}

\section{Article history:}

Received 15 October 2008

Accepted 27 November 2008

Available online 6 December 2008

\section{Keywords:}

Torsional NEMS actuator

Capillary force

Instability

Bifurcation

\begin{abstract}
A B S T R A C T
The static and dynamic instabilities of a torsional MEMS/NEMS actuator caused by capillary effects are studied, respectively. An instability number, $\eta$, is defined, and the critical gap distance, $g_{c r}$, between the mainplate and the substrate is derived. According to the values of $\eta$ and $g$, the instability criteria of the actuator are presented. The dimensionless motion equation of the MEMS/NEMS torsional actuator is derived when it makes nonlinear oscillation under capillary force. The qualitative analysis of the nonlinear equation is made, and the phase portraits are presented on the phase plane. In addition, the bifurcation phenomena in the system are also analyzed.
\end{abstract}

(c) 2008 Elsevier Inc. All rights reserved.

\section{Introduction}

Torsional actuators have broad applications in the microelectro-mechanical systems (MEMS) and nano-electro-mechanical systems (NEMS) devices [1-4], such as torsional radio frequency (RF) switches, tunable torsional capacitors, torsional micromirrors, etc. Torsional actuators, like parallel plate actuators, are often fabricated to be with suspended structures which have a minimum gap distance and a large area between the moveable plate and the underlying substrate. Under the condition, some surface and intermolecular forces become very important due to the large surface-to-volume ratio and the small gap distance [5]. As a result, the structures will lose their stability, and the moveable plate could snap down and permanently adhere to their substrates due to the actions of the surface forces.

The surface forces which play important roles in the MEMS and NEMS devices include van der Waals (vdW) force, Casimir force and capillary force, etc. The vdW and Casimir forces are electromagnetic in nature, and the Casimir force can be regarded as the retarded vdW force [6-9]. And they always exist between two objects close to each other. The influence of vdW and Casimir forces on the stability of the MEMS and NEMS actuators with suspended structures has been extensively reported in many literatures [6-19]. Chan et al. [10] showed that Casimir force can be used to actuate the NEMS devices under certain condition. Lin and Zhao [13-15] studied the influence of Casimir force on the nanoscale electrostatic parallel plate RF switch. They found that the pull-in and adhesion [13] could be induced by Casimir force

\footnotetext{
* Corresponding author. Fax: +1 861062561284 .

E-mail address: yzhao@imech.ac.cn (Y.-P. Zhao).
}

when the ratio of the gap to the length was sufficiently small. They also studied the dynamic behavior of the parallel plate NEMS RF switches with the consideration of the vdW effects [14]. Guo and Zhao [16] studied the inherent instability of an electrostatic torsional NEMS actuator with the vdW and Casimir effects taken into consideration, and derived the critical conditions when the pull-in phenomena of the actuator occurred. The influence of vdW force on dynamic stability of an electrostatic torsional NEMS actuator was also studied [17], and the bifurcation behaviors were analyzed. Gusso and Delben [18] performed a theoretical analysis focusing on the effect of the Casimir force on the pull-in parameters of electrostatic torsional actuators made from silicon. Ramezani and coworkers [19] investigated the two-point boundary value problem (BVP) of the nano-cantilever deflection subjected to Casimir and electrostatic forces, and derived the instability point of the nanobeam by using analytical and numerical methods.

Capillary force occurs between two objects when the liquid bridge forms between them [20]. It is found that even for the lowest attainable relative humidity the capillary forces are still present [21]. Suspended structures are typically made by forming a layer of the plate or beam material on top of a sacrificial layer of another material and wet etching the sacrificial layer. Thus it is easy for developing capillary force in the rinse-dry cycle following the wet removal of a sacrificial layer as the rinse solution is gradually removed. If the capillary force is large enough, the structure will collapse and adhere to the substrate. So it is very important to study the instability conditions of the MEMS and NEMS devices under capillary forces [22]. The mechanical stability and adhesion phenomena of a parallel plate MEMS actuator were studied by Mastrangelo and Hsu $[23,24]$ with the influence of capillary force taken into consideration. But there are few liter- 


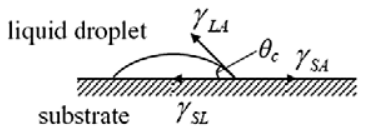

(a)

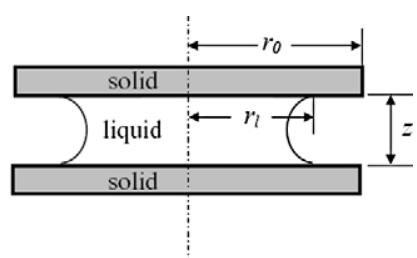

(b)
Fig. 1. (a) Contact angle at a solid-liquid interface. (b) Liquid bridge between two parallel circular plates.

atures about the stability of a torsional MEMS or NEMS actuator under capillary forces. In the paper, the simplified one-degree-offreedom (1DOF) model is applied to study the static and dynamic instability of a torsional MEMS/NEMS actuator, which is caused by the capillary effects. And the instability criteria of the actuator and the phase portraits of nonlinear oscillation will be presented.

\section{The capillary torque in a torsional MEMS/NEMS actuator}

For a drop of liquid placed on the surface of a solid as shown in Fig. $1 \mathrm{a}$, the contact angle $\theta_{\mathrm{c}}$ is determined by the balance among the liquid-air (LA), solid-air (SA), and solid-liquid (SL) interfacial tensions, which are denoted by $\gamma_{\mathrm{LA}}, \gamma_{\mathrm{SA}}$ and $\gamma_{\mathrm{SL}}$, respectively. At equilibrium, these tensions satisfy Young's equation [25-28]

$\gamma_{\mathrm{SA}}=\gamma_{\mathrm{SL}}+\gamma_{\mathrm{LA}} \cos \theta_{\mathrm{C}} \quad\left(0<\theta_{\mathrm{c}}<\pi\right)$.

At the equilibrium state, the energy of the system in Fig. 1b is [29]

$U_{S}=U_{S_{0}}-2 \gamma_{1} \pi r_{1}^{2} \cos \theta_{\mathrm{c}}$,

where $U_{S_{0}}$ is a constant, $\gamma_{1}$ the surface energy of liquid, and $\gamma_{1}=\gamma_{\mathrm{LA}}$.

The force $F$ is related to the interfacial energy by

$F=-\left(\frac{d U_{S}}{d z}\right)=-\left(\frac{d U_{S}}{d r_{1}}\right) \cdot\left(\frac{d r_{1}}{d z}\right)$.

Since the volume of the drop, $V_{1}=2 \pi r_{1}^{2} z$, is constant, then $d r_{1} / d z=-r_{1} / 2 z$. Thus the capillary pressure, $q$, exerted on the rigid plate is

$q=-\frac{F}{\pi r_{1}^{2}}=\frac{2 \gamma_{1} \cos \theta_{c}}{z}$.

The pressure is proportional to the term $\gamma_{1} \cos \theta_{c}$, known as the adhesion tension. Pressure $q$ is attractive or repulsive depending on the sign of $\cos \theta_{c}$ and is nonlinear with respect to the spacing $z$ with a singularity at $z=0$.

A torsional MEMS/NEMS actuator mainly consists of a mainplate, a torsional beam and a substrate (as shown in Fig. 2). The schematic of its 1DOF model is shown in Fig. 3, where the mainplate of the device is considered to be a tiltable rigid body in a first-order approximation. Hence, the angle of torsion $\varphi$ is the only variable in the model (shown as Fig. 3). When the mainplate rotates anti-clockwise an angle $\varphi$, the capillary differential forces acting on an arbitrary infinitesimal area $d S$ (as shown in Fig. 2) at the both sides of torsional beam are, respectively,

$d F_{\text {cap }}^{\mathrm{L}}=\frac{2 \gamma_{1} \cos \theta_{\mathrm{c}}}{d-r \sin \varphi} \cdot r d r d \theta$ and $d F_{\text {cap }}^{\mathrm{R}}=\frac{2 \gamma_{1} \cos \theta_{\mathrm{c}}}{d+r \sin \varphi} \cdot r d r d \theta$,

where $\theta_{c}$ is the contact angle of liquid-solid interface, and $d$ is the gap distance between mainplate and substrate. So the torque of two capillary differential forces to fixed torsional beam can be derived as
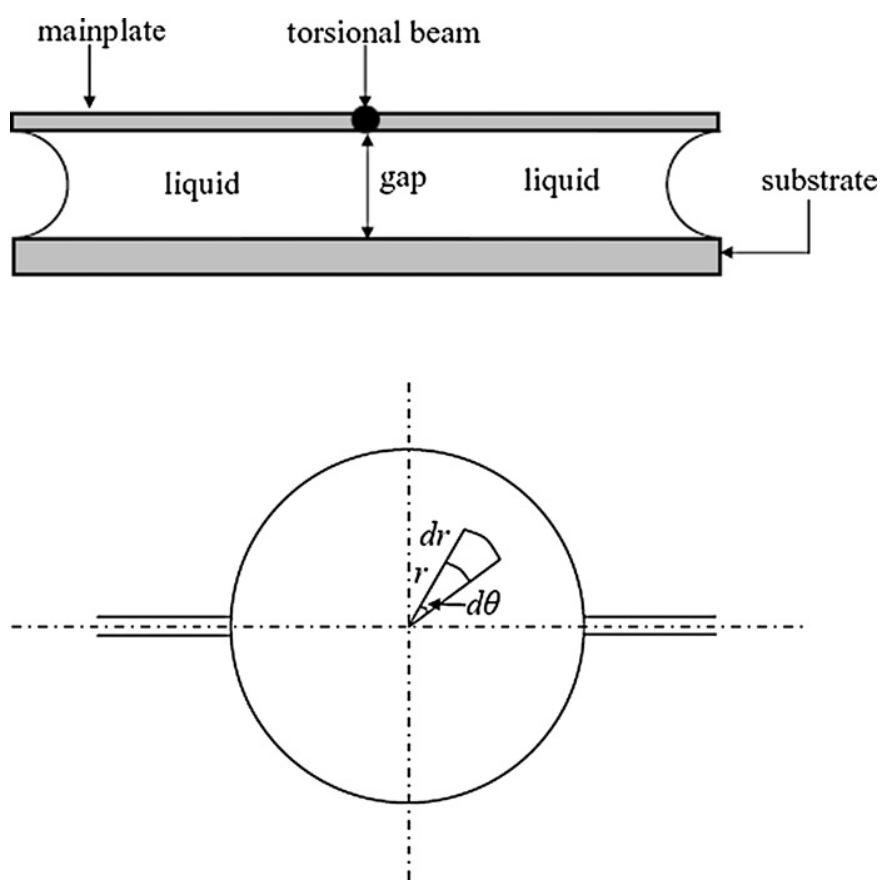

Fig. 2. Schematic side view and planform of a torsional NEMS actuator.

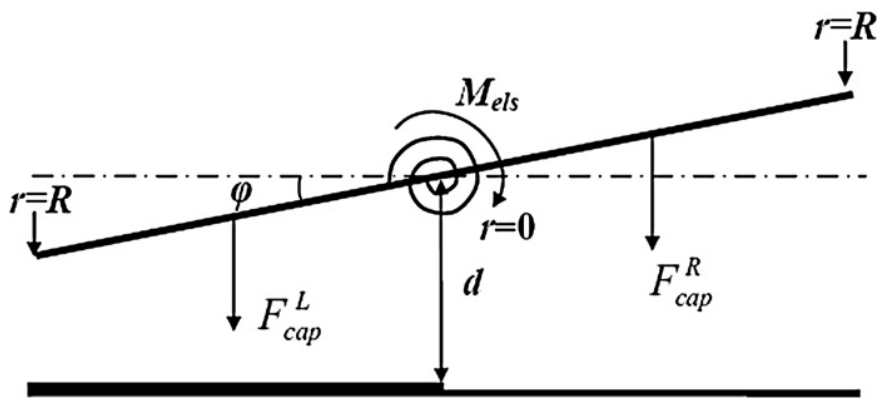

Fig. 3. 1DOF model of torsional actuator with capillary force.

$$
\begin{aligned}
M_{\text {cap }} & =\int\left(d F_{\text {cap }}^{\mathrm{L}}-d F_{\text {cap }}^{\mathrm{R}}\right) \cdot r \\
& =-\frac{2 \pi \gamma_{1} \cos \theta_{\mathrm{c}}}{\sin \varphi} \cdot\left[R^{2}+\frac{d^{2}}{\sin ^{2} \varphi} \cdot \log \left(1-\frac{R^{2} \sin ^{2} \varphi}{d^{2}}\right)\right],
\end{aligned}
$$

where $R$ is the radius of the mainplate.

Due to $d / R \ll 1$, the tilting angle is small, and an approximation is used, i.e., $\sin \varphi \approx \varphi$. Therefore, the maximum tilting angle is $\varphi_{0} \approx \sin \varphi_{0}=d / R$. Furthermore, the normalized tilting angle, $\beta=\varphi / \varphi_{0}$, is introduced, where the value of $\beta$ is in the range of 0 and 1 . Thus Eq. (6) can be rewritten as

$M_{\text {cap }}=-\frac{S \gamma_{1} \cos \theta_{\mathrm{c}}}{g} \cdot\left[\frac{2}{\beta}+\frac{1}{\beta^{3}} \cdot \log \left(1-\beta^{2}\right)^{2}\right]$,

where $S=\pi R^{2}$ is the area of mainplate, and $g$ is a dimensionless gap distance, which equals to $d / R$.

When the mainplate rotates around the torsional beam, a restoring torque will be produced. The torque is caused by elastic restoring force of the beam, which can be simplified as a flexure spring with torsional stiffness, $K$. Then elastic restoring torque of the torsional beam can be expressed as

$M_{\mathrm{els}}=K \varphi=K g \beta=2 \frac{G I_{\rho} g}{l} \beta$,

where $G, I_{\rho}, l$, respectively, denotes the shear modulus, the polar moment of inertia and the length of the torsional beam. The polar 


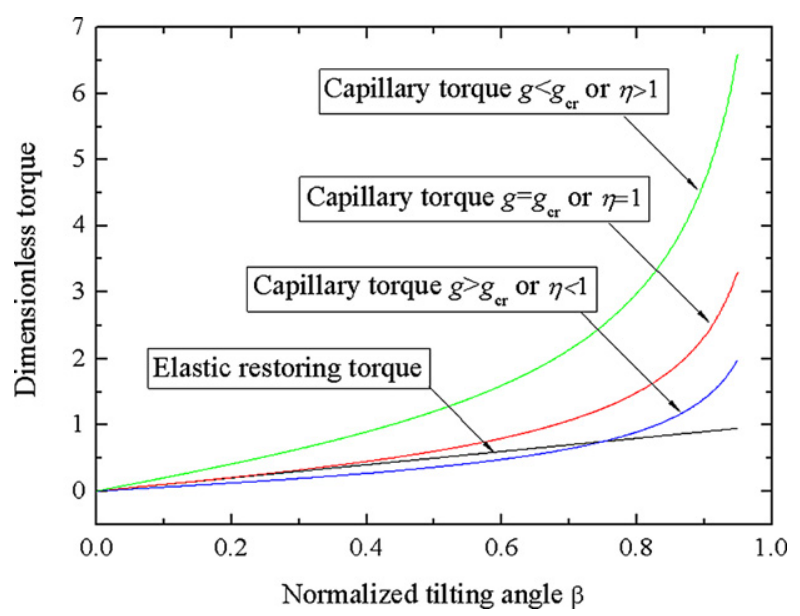

Fig. 4. The relations of the dimensionless capillary torque and the elastic restoring torque for the different values of the instability number and the dimensionless gap.

moment of inertia, $I_{\rho}$, of the circular cross-section is $I_{\rho}=\pi r^{4} / 2$, where $r$ is the radius of the torsional beam.

\section{Static instability caused by capillary torque}

For the system in Fig. 3, the stability condition is the equilibrium between capillary torque and elastic restoring torque of torsional beam, i.e., $M_{\text {cap }}=M_{\text {els }}$. So the equilibrium equation of the system can be derived, and its dimensionless form is

$\beta+\eta \cdot\left[\frac{2}{\beta}+\frac{1}{\beta^{3}} \cdot \log \left(1-\beta^{2}\right)^{2}\right]=0$,

where $\eta$ is a dimensionless quantity, and it is the ratio between the capillary torque and elastic restoring torque. $\eta=\Gamma \cdot f$, where the dimensionless quantity $\Gamma$ equals to $S \gamma_{1} / K$, which is the ratio between the surface energy and elastic energy, and $f=\cos \theta_{c} / g^{2}$, which is a shape factor.

It can be derived from Eq. (9) that the expression of $\eta$ is

$\eta=-\frac{\beta^{4}}{2 \beta^{2}+\log \left(1-\beta^{2}\right)^{2}} \quad(0<\beta<1)$.

The critical value of $\eta$ can be calculated by

$\eta_{\mathrm{cr}}=\lim _{\beta \rightarrow 0} \eta=1$.

Furthermore, according to $\eta=\Gamma \cdot f$, we can derive the critical gap distance of instability

$g_{\mathrm{cr}}=\sqrt{\frac{S \gamma_{1}}{K} \cos \theta_{\mathrm{c}}}$

We can define the dimensionless quantity, $\eta$, as an instability number. When $\eta>1$, i.e., the gap distance $g<g_{\mathrm{cr}}$, the capillary torque is always greater than the elastic restoring torque, and the torsional actuator is unstable. An arbitrary small angle perturbation of the torsional actuator can lead to the pull-in and collapse of the structure. On the contrary, when $\eta<1$, i.e., the gap distance $g>g_{\text {cr }}$, the capillary torque is smaller than the elastic restoring torque. The system can not lose its stability until the tilting angle reaches a certain value. These results can be intuitively illustrated in Fig. 4. The values of geometric and physical parameters in the calculation are listed in Table 1 . The value of the tilting angle is dependent on the gap distance. The function relation of $d$ and $\beta$ can be derived by Eq. (10), which is illustrated in Fig. 5.

In Ref. [16], the influence of the vdW and Casimir effects on the inherent instability of an electrostatic torsional NEMS actuator was studied. We can compare the vdW and Casimir effects with
Table 1

Parameters of numerical calculation.

\begin{tabular}{lll}
\hline Items & Symbol & Value \\
\hline Surface energy & $\gamma_{1}$ & $7.2 \times 10^{-2} \mathrm{Nm}^{-1}$ \\
Hamaker constant & $\mathrm{A}$ & $(0.4-4) \times 10^{-19} \mathrm{~J}$ \\
Reduced Planck's constant & $\hbar$ & $1.055 \times 10^{-34} \mathrm{Js}$ \\
Speed of light & $c$ & $2.998 \times 10^{8} \mathrm{~m} \mathrm{~s}^{-1}$ \\
Shear modulus & $G$ & $6.6 \times 10^{10} \mathrm{~Pa}$ \\
Contact angle & $\theta_{\mathrm{c}}$ & $5 \pi / 12$ \\
Radius of rotational plate & $R$ & $10^{-4} \mathrm{~m}$ \\
Radius of torsional beam & $r$ & $2 \times 10^{-6} \mathrm{~m}$ \\
Length of torsional beam & $l$ & $5 \times 10^{-5} \mathrm{~m}$ \\
\hline
\end{tabular}

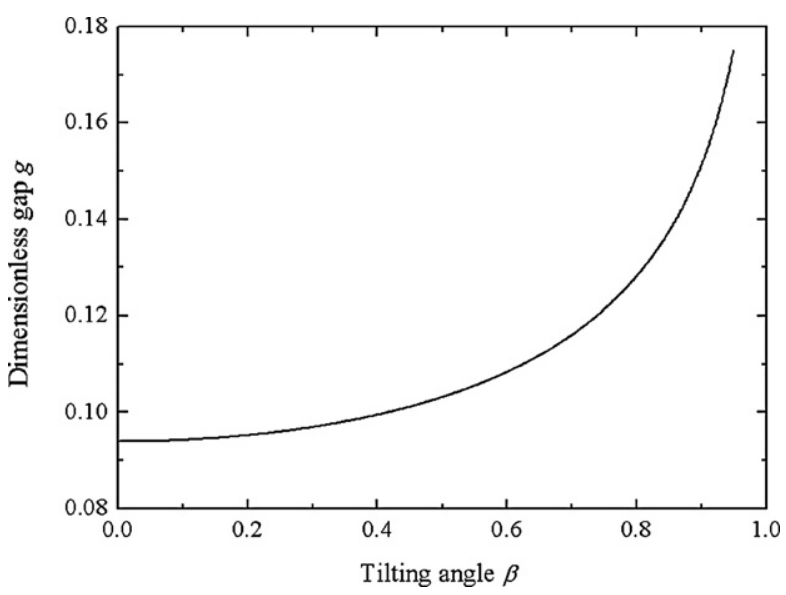

Fig. 5. Variation of the dimensionless gap distance with the tilting angle.

capillary effects. The vdW torque and Casimir torque expressed by normalized tilting angle can be derived as [11], respectively,

$M_{\mathrm{vdW}}=\frac{A}{6 g^{3}} \cdot \frac{1}{\beta^{3}} \cdot\left[\frac{\beta^{2}\left(3 \beta^{2}-1\right)}{\left(1-\beta^{2}\right)^{2}}-\log \left(1-\beta^{2}\right)\right]$,

$M_{\text {Casimir }}=\frac{\pi^{3} \hbar c}{360 R} \cdot \frac{1}{g^{4}} \cdot \frac{\beta\left(3+\beta^{2}\right)}{\left(1-\beta^{2}\right)^{3}}$,

where $A=\pi^{2} C \rho^{2}$ is the Hamaker constant, which lies in the range $(0.4-4) \times 10^{-19} \mathrm{~J}, \hbar$ is the Planck's constant divided by $2 \pi$, which is equal to $1.055 \times 10^{-34} \mathrm{Js}$, and $c$ is the speed of light and equals to $2.998 \times 10^{8} \mathrm{~ms}^{-1}$.

Introduce two dimensionless quantities, $M_{\text {cap } / v d W}$ and $M_{\text {cap/Casimir }}$,

$M_{\text {cap } / \mathrm{vdW}}=\lim _{\beta \rightarrow 0} \frac{M_{\mathrm{cap}}}{M_{\mathrm{vdW}}}=\frac{4 S \gamma_{1} \cos \theta_{\mathrm{c}}}{A} \cdot g^{2}$,

$M_{\text {cap } / \text { Casimir }}=\lim _{\beta \rightarrow 0} \frac{M_{\text {cap }}}{M_{\text {Casimir }}}=\frac{120 S R \gamma_{1} \cos \theta_{c}}{\pi^{3} \hbar c} \cdot g^{3}$,

where $M_{\mathrm{cap} / \mathrm{vdW}}$ is the ratio between capillary torque and vdW torque, and $M_{\text {cap/Casimir }}$ is the ratio between capillary torque and Casimir torque. The logarithm function curves of two dimensionless torques are shown in Fig. 6. It can be shown that capillary torque has a much longer range than the vdW and Casimir torques.

\section{Dynamic instability caused by capillary torque}

If the dynamic effects are considered in the process of the rotation, the mainplate will make nonlinear oscillation. In the section, the phase portraits of the nonlinear oscillation will be presented, and the bifurcation behaviors of the system will be analyzed. With the inertial and damping effects taken into consideration, there exist an inertia torque and a damping torque in the model,

$M_{\text {interia }}=I \ddot{\varphi}=I g \ddot{\beta} \quad$ and $\quad M_{\text {damping }}=C \dot{\varphi}=C g \dot{\beta}$, 


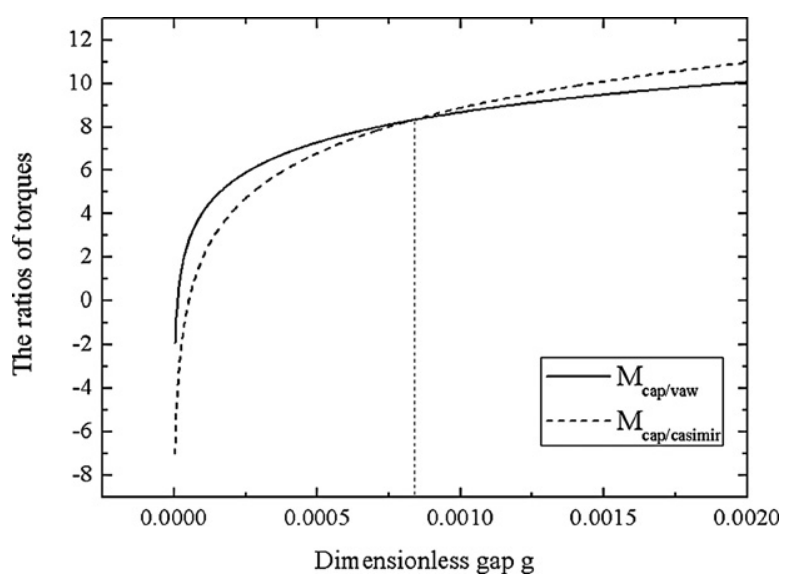

Fig. 6. The logarithm function curves of $M_{\mathrm{cap} / \mathrm{vdW}}$ (the ratio between capillary torque and vdW torque) and $M_{\mathrm{cap} / \text { Casimir }}$ (the ratio between capillary torque and Casimir torque).

where $C$ and $I$ are the effective damping constant and inertia moment of the mainplate, respectively.

When the oscillation occurs, the dimensionless equation of motion including capillary torque can be written as

$\frac{d^{2} \beta}{d \tau^{2}}+\mu \frac{d \beta}{d \tau}+\omega \beta=-\xi\left(\frac{1}{\beta}+\frac{1}{\beta^{3}} \cdot \log \left(1-\beta^{2}\right)\right)$

where the dimensionless quantities are

$\mu=\frac{C T}{I}, \quad \omega=\frac{K T^{2}}{I}, \quad \xi=\frac{2 S \gamma_{1} T^{2} \cos \theta_{\mathrm{c}}}{I g^{2}}, \quad \tau=\frac{t}{T}$,

and $T$ is a characteristic time. Equation (18) is a second-order nonlinear and inhomogeneous ordinary differential equation. The qualitative analysis of the nonlinear equation is made below to obtain the whole properties of the solutions.

Setting $d \beta / d \tau=\psi$, Eq. (18) can be transformed into the following autonomous system

$$
\left\{\begin{array}{l}
\frac{d \beta}{d \tau}=\psi, \\
\frac{d \psi}{d \tau}=-\mu \psi-\omega \beta-\xi\left(\frac{1}{\beta}+\frac{1}{\beta^{3}} \cdot \log \left(1-\beta^{2}\right)\right) .
\end{array}\right.
$$

The Jacobian matrix of the autonomous system is

$$
\left(\begin{array}{cc}
0 & 1 \\
F^{\prime}(\beta) & -\mu
\end{array}\right)
$$

where

$F(\beta)=-\omega \beta-\xi\left(\frac{1}{\beta}+\frac{1}{\beta^{3}} \cdot \log \left(1-\beta^{2}\right)\right)$,

and the prime denotes the differentiation with respect to $\beta$. The corresponding eigenvalues of the Jacobian matrix are

$\lambda_{1,2}=\frac{1}{2}\left(-\mu \pm \sqrt{\mu^{2}+4 F^{\prime}(\beta)}\right)$.

The equilibrium states of the autonomous system depend on the solutions of the equation $F(\beta)=0$. According to the values of the instability number $\eta$ and the dimensionless gap distance $g$, we can discuss the equilibrium states of the autonomous system by following three cases:

(i) When $\eta<1$, i.e., $g>g_{\text {cr }}$.

For a specified value of the dimensionless gap $g$ satisfying the condition, the equation $F(\beta)=0$ has two real roots with the

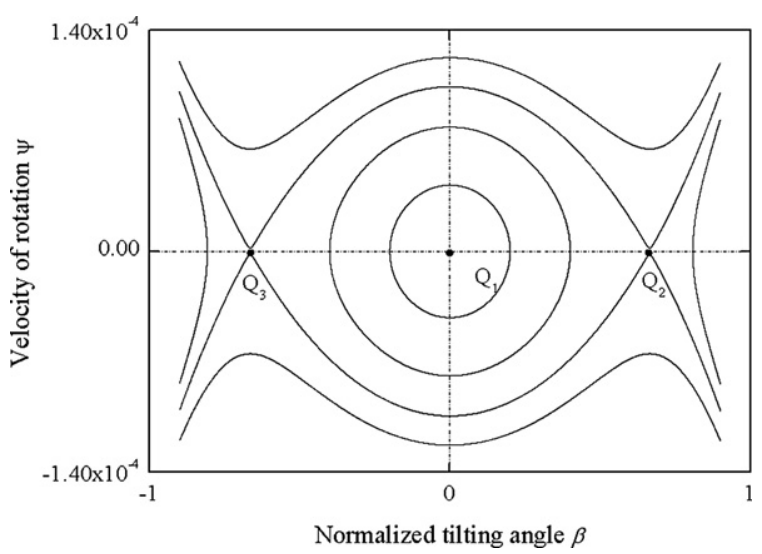

Fig. 7. The phase portrait on the phase plane without the damping effects: periodic orbits and homoclinic orbits.

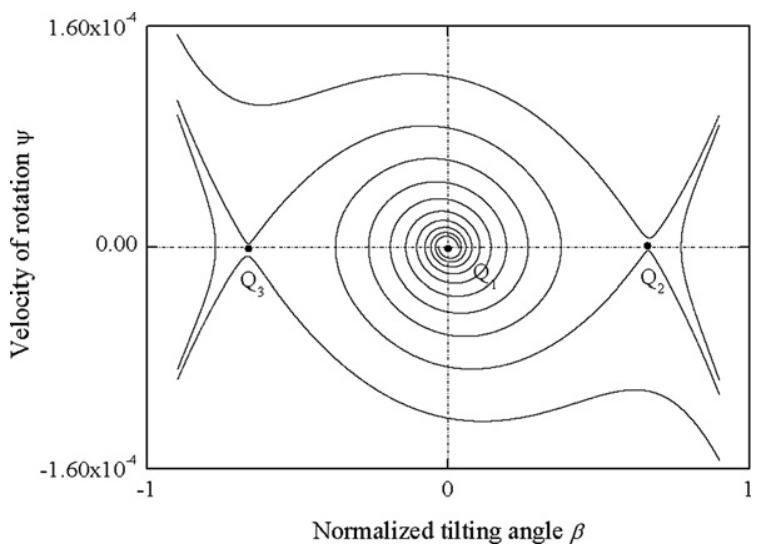

Fig. 8. The phase portrait on the phase plane with the damping effects taken into consideration: heteroclinic orbits.

variable $\beta$ in the range of $[0,1)$. They are $\beta_{1}=0$ and $\beta_{2}$, respectively. So the autonomous system has at least two equilibrium points $Q_{1}(0,0), Q_{2}\left(\beta_{2}, 0\right)$. Since the system has a symmetric structure, and the load of capillary torque is antisymmetric, there exists the third equilibrium point $Q_{3}\left(-\beta_{2}, 0\right)$ in the system.

The equilibrium point $Q_{1}$ is a center point when the parameter $\mu=0$, and is a stable focus point when the parameter $\mu \neq$ 0 . It can be concluded that the mainplate makes convergent oscillation near the focus point because of the damping, and makes periodic oscillation if the damping is neglected. The equilibrium point $Q_{2}$ and $Q_{3}$ are unstable saddle points for any $\mu$. The phase orbits connecting three equilibrium points on the phase plane are shown in Figs. 7 and 8, which include the periodic, heteroclinic and homoclinic orbits. In addition, to equilibrium point $Q_{1}$, because the eigenvalues of the Jacobian matrix are a couple of pure imaginary roots when the parameter $\mu=0$, the point $(\beta, \psi ; \mu)=(0,0 ; 0)$ is a Hopf bifurcation point.

(ii) When $\eta=1$, i.e., $g=g_{\text {cr }}$.

When the dimensionless gap $g$ approaches the critical value $g_{\text {cr }}$, the point of intersection $Q_{2}$ tends towards the point $Q_{1}$, and they finally converge at the point $Q_{1}(0,0)$, which satisfies simultaneously the equation $F(\beta)=0$ and the equation $F^{\prime}(\beta)=0$. The eigenvalues of corresponding Jacobian matrix are two repeated zero roots when the parameter $\mu=0$, and a zero root and a nonzero real root when $\mu \neq 0$. In addition, the nonzero real root is negative if the parameter $\mu>0$, and is positive if $\mu<0$. So when the dimensionless gap $g$ reaches 
critical value $g_{\mathrm{cr}}$, the equilibrium point $(\beta, \psi ; \mu)=(0,0 ; 0)$ becomes a fork bifurcation point.

(iii) When $\eta>1$, i.e., $g<g_{\text {cr }}$.

The structure is instability, and the pull-in will automatically happen when $\eta>1$, i.e., $g<g_{\text {cr }}$. So the autonomous system has no equilibrium point in the case.

\section{Summary}

The static and dynamic instabilities of a torsional MEMS/NEMS actuator caused by capillary effects are studied in the present paper. Due to the action of capillary effects, the torsional MEMS/ NEMS actuator can be inherently instable. In certain condition, the actuators can collapse and permanently adhere to their substrates in the process of fabrication. An instability number $\eta$ is defined in the paper, and the critical gap distance $g_{c r}$ between the mainplate and the substrate is derived. And then the instability criteria are presented.

The dynamics analysis of the torsional NEMS actuator shows that it makes nonlinear oscillation under capillary torque. The dimensionless motion equation of the nonlinear oscillation is presented. The qualitative analysis of the nonlinear equation shows that the equilibrium points of its corresponding autonomous system include stable focus point, center point, and unstable saddle point. The phase portraits show the periodic, heteroclinic and homoclinic orbits. There are bifurcation phenomena in the nonlinear system, and the Hopf bifurcation point and the fork bifurcation point are presented, respectively.

\section{Acknowledgments}

This work was supported by the National Natural Science Foundation of China (NSFC, Grant no. 10802057), National Basic Research Program of China (973 Program, Grant no. 2007CB310500),
National High-tech R\&D Program of China (863 Program, Grant no. 2007AA04Z348), and National Natural Science Foundation of China (NSFC, Grant nos. 10772180 and 10721202).

\section{References}

[1] F. Plötz, S. Michaelis, R. Aigner, H.-J. Timme, J. Binder, R. Noé, Sens. Actuators A Phys. 92 (2001) 312.

[2] J.W. Hsieh, W.L. Fang, Sens. Actuators A Phys. 79 (2000) 64.

[3] X.M. Zhang, F.S. Chau, C. Quan, Y.L. Lam, A.Q. Liu, Sens. Actuators A Phys. 90 (2001) 73.

[4] H.G. Craighead, Science 290 (2000) 1532.

[5] Y.P. Zhao, L.S. Wang, T.X. Yu, J. Adhes. Sci. Technol. 17 (2003) 519.

[6] W.H. Lin, Y.P. Zhao, Chaos Solitons Fractals 23 (2005) 1777.

[7] G. Palasantzas, J. Appl. Phys. 101 (2007) 063548.

[8] R. Esquivel-Sirvent, L. Reyes, J. Barcenas, New J. Phys. 8 (2006) 241.

[9] R.C. Batra, A. Porfiri, D. Spinello, J. Sound Vibration 315 (2008) 939.

[10] H.B. Chan, V.A. Aksyuk, R.N. Kleiman, D.J. Bishop, F. Capasso, Science 291 (2001) 194.

[11] R. Esquivel-Sirvent, J. Appl. Phys. 102 (2007) 034307.

[12] F. Pinto, J. Phys. A Math. Theoret. 41 (2008) 164033.

[13] W.H. Lin, Y.P. Zhao, Microsyst. Technol. 11 (2005) 90.

[14] W.H. Lin, Y.P. Zhao, Chin. Phys. Lett. 20 (2003) 2070.

[15] W.H. Lin, Y.P. Zhao, J. Phys. D Appl. Phys. 40 (2007) 1649.

[16] J.G. Guo, Y.P. Zhao, J. Microelectromech. Syst. 13 (2004) 1027.

[17] J.G. Guo, Y.P. Zhao, Int. J. Solids Struct. 43 (2006) 675.

[18] A. Gusso, G.J. Delben, Sens. Actuators A Phys. 135 (2007) 792.

[19] A. Ramezani, A. Alasty, J. Akbari, Microsyst. Technol. 14 (2008) 145.

[20] Z. Wei, Y.P. Zhao, J. Phys. D Appl. Phys. 40 (2007) 4368.

[21] P.J. van Zwol, G. Palasantzas, J.Th.M. De Hosson, Phys. Rev. E 78 (2008) 031606.

[22] G. Palasantzas, J. Appl. Phys. 101 (2007) 053512.

[23] C.H. Mastrangelo, C.H. Hsu, J. Microelectromech. Syst. 2 (1993) 33.

[24] C.H. Mastrangelo, C.H. Hsu, J. Microelectromech. Syst. 2 (1993) 44.

[25] R.S. Burdon, Surface Tension and the Spreading of Liquids, Cambridge Univ. Press, Cambridge, 1949.

[26] J.T. Davies, E.K. Rideal, Interfacial Phenomena, Academic Press, New York, 1963.

[27] P.C. Hiemenz, Principles of Colloid and Surface Chemistry, Marcel Dekker, New York, 1986.

[28] A.W. Adamson, Physical Chemistry of Surfaces, Wiley, New York, 1990

[29] M.A. Fortes, J. Colloid Interface Sci. 88 (1982) 338. 